\title{
Antifungal Cyclopentenediones from Piper coruscans
}

Xing-Cong Li, ${ }^{* \dagger}$ Daneel Ferreira, ${ }^{\dagger}$ Melissa R. Jacob, ${ }^{\dagger}$ Qifeng Zhang, ${ }^{\dagger}$ Shabana I. Khan, ${ }^{\dagger}$ Hala N. ElSohly, ${ }^{\dagger}$ Dale G. Nagle, ${ }^{\ddagger}$ Troy J. Smillie, ${ }^{\dagger}$ Ikhlas A. Khan, ${ }^{\dagger, \ddagger}$ Larry A. Walker, ${ }^{\dagger, \S}$ and Alice M. Clark ${ }^{*, \dagger, \dagger, \perp}$

\section{Table of contents}

1. Extraction and isolation of coruscanones A (1) and B (2)

2. NMR data of coruscanones A (1) and B (2)

3. Biological activity data of coruscanones A (1) and B (2)

4. Synthesis of coruscanones A (1) and B (2)

5. $300 \mathrm{MHz}{ }^{1} \mathrm{H}$ NMR spectrum of coruscanone $\mathrm{A}(\mathbf{1})$ in $\mathrm{C}_{6} \mathrm{D}_{6}(\mathbf{1 a} / \mathbf{1} \mathbf{b}: \sim 5 / 1)$

6. $300 \mathrm{MHz}{ }^{1} \mathrm{H}$ NMR spectrum of coruscanone $\mathrm{A}(\mathbf{1})$ in $\mathrm{C}_{6} \mathrm{D}_{6}(\mathbf{1 a} / \mathbf{1} \mathbf{b}: \sim 1 / 1)$ after sitting in the NMR tube at r.t. for $24 \mathrm{hrs}$

7. $300 \mathrm{MHz}{ }^{1} \mathrm{H}$ NMR spectrum of coruscanone $\mathrm{A}(\mathbf{1})$ in $\mathrm{CDCl}_{3}(\mathbf{1 a} / \mathbf{1} \mathbf{b}: \sim 1 / 1)$ after sitting in the NMR tube at r.t. for $12 \mathrm{hrs}$

8. $400 \mathrm{MHz}{ }^{1} \mathrm{H}$ NMR spectrum of synthetic coruscanone $\mathrm{A}(\mathbf{1})$ in $\mathrm{CDCl}_{3}(\mathbf{1 a} / \mathbf{1} \mathbf{b}: \sim 1 / 1)$ after sitting in the NMR tube at r.t. for $12 \mathrm{hrs}$

9. $300 \mathrm{MHz}{ }^{1} \mathrm{H}$ NMR spectrum of coruscanone $\mathrm{B}(\mathbf{2})$ in $\mathrm{CDCl}_{3}(\mathbf{2 a} / \mathbf{2} \mathbf{b}: ~ 1 / 1.2)$ after sitting in the NMR tube at r.t. for $24 \mathrm{hr}$

10. $400 \mathrm{MHz}{ }^{1} \mathrm{H}$ NMR spectrum of synthetic coruscanone B (2) in $\mathrm{CDCl}_{3}$ (predominant $2 \mathbf{b}$ ) at r.t.

11. $400 \mathrm{MHz}{ }^{1} \mathrm{H}$ NMR spectrum of synthetic coruscanone $\mathrm{B}(\mathbf{2})$ in $\mathrm{CDCl}_{3}(\mathbf{2 b} / \mathbf{2 a}$ : 2.4:1) after sitting in the NMR tube at r.t for $5 \mathrm{hr}$.

12. $400 \mathrm{MHz}{ }^{1} \mathrm{H}$ NMR spectrum of 4 -ylidenebutenolide 5 in $\mathrm{CDCl}_{3}$ 
The plant material was collected by Mr. Manuel Rimachi in Loreto, Peru in April, 1996 and identified by Mr. M. Rimachi and Dr. Sidney McDaniel. A voucher specimen of this plant is deposited at the Herbarium of Mississippi State University (Voucher \# IBE 11553).

The dried root-stem-leave (468 g) of Piper coruscans was ground to a coarse powder and percolated with $95 \%$ EtOH. Removal of the solvent under vacuum yielded an EtOH extract (15.2 g), a portion $(5 \mathrm{~g})$ of which was directly applied to a silica gel column using a mixture of hexane-CHCl $\mathrm{CH}_{3}$ as solvents. Two consecutive fractions eluted by hexane- $\mathrm{CHCl}_{3}(1: 1)$, which are the most active against $C$. albicans $\left(\mathrm{IC}_{50}<2 \mu \mathrm{g} / \mathrm{mL}\right)$, were individually chromatographed on reversed-phase silica gel $\left(\mathrm{C}_{18}\right)$ using $60 \% \mathrm{CH}_{3} \mathrm{CN}-\mathrm{H}_{2} \mathrm{O}$ as solvent to afford coruscanones $\mathrm{A}(1,98 \mathrm{mg})$ and $\mathrm{B}(2,135 \mathrm{mg})$, respectively.

Coruscanone A (1): yellow powder, mp. $86^{\circ} \mathrm{C}$; UV (MeOH), $\lambda_{\max }(\log \varepsilon) 232$ (4.45), 348 (4.58) $\mathrm{nm}$; IR $(\mathrm{NaCl}) v_{\max }, 1666,1615,1547,1444,1306,1177,1034,973,874,765,692 \mathrm{~cm}^{-1}$; NMR data, see Tables 3 and 4; HRESIMS $m / z[\mathrm{M}+\mathrm{H}]^{+} 255.1016$ (calcd for $\mathrm{C}_{16} \mathrm{H}_{15} \mathrm{O}_{3}, 255.1016$ ), $[\mathrm{M}+\mathrm{Na}]^{+} 277.0828$ (calcd for $\mathrm{C}_{16} \mathrm{H}_{14} \mathrm{O}_{3} \mathrm{Na}, 277.0835$ ).

Coruscanone B (2): yellow needles, mp. $124^{\circ} \mathrm{C}$; UV (MeOH), $\lambda_{\max }(\log \varepsilon) 232$ (4.24), 348 (4.40) $\mathrm{nm}$; IR $(\mathrm{NaCl}) v_{\max }, 1708,1648,1630,1588,1448,1353,1016,978,904,877,704,690 \mathrm{~cm}^{-1}$; NMR data, see Tables 3 and 4; HRESIMS $m / z[\mathrm{M}+\mathrm{H}]^{+} 241.0849$ (calcd for $\mathrm{C}_{15} \mathrm{H}_{13} \mathrm{O}_{3}, 241.0859$ ), [M+ Na $]^{+} 263.0660$ (calcd for $\mathrm{C}_{15} \mathrm{H}_{12} \mathrm{O}_{3} \mathrm{Na}, 263.0678$ ).
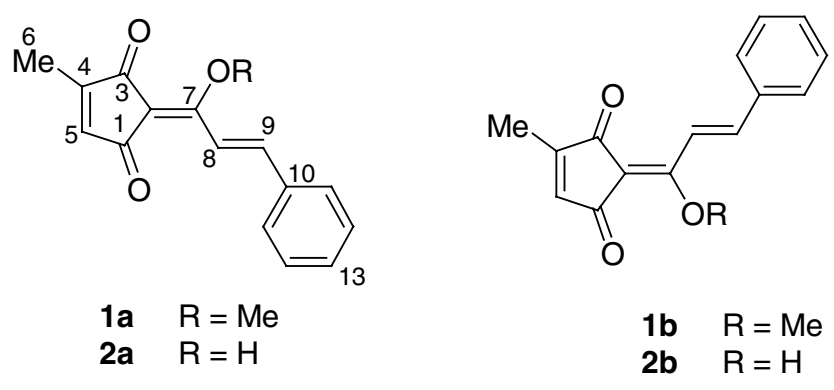
Table 3. ${ }^{13} \mathrm{C}$ NMR data for compounds $\mathbf{1}$ and $\mathbf{2}(\boldsymbol{\delta}$, ppm)

\begin{tabular}{rrrrr}
\hline $\mathbf{C}$ & $\mathbf{1 a}^{a}$ & $\mathbf{1 b}^{a}$ & $\mathbf{2 a}^{b}$ & $\mathbf{2 b}^{b}$ \\
\hline 1 & 191.1 & 193.8 & 191.8 & 200.7 \\
2 & 109.4 & 109.5 & 103.5 & 103.2 \\
3 & 195.0 & 192.5 & 201.3 & 192.2 \\
4 & 156.4 & 156.9 & 154.1 & 158.1 \\
5 & 140.9 & 140.6 & 140.8 & 137.1 \\
6 & 10.9 & 11.0 & 10.7 & 11.5 \\
7 & 168.5 & 168.3 & 167.7 & 167.9 \\
8 & 121.1 & 121.3 & 117.7 & 117.5 \\
9 & 142.4 & 142.4 & 143.3 & 143.2 \\
10 & 136.1 & 136.2 & 134.9 & 134.9 \\
11 & 128.8 & 128.8 & 128.8 & 128.7 \\
12 & 129.3 & 129.3 & 129.0 & 129.0 \\
13 & 130.4 & 130.4 & 130.8 & 130.8 \\
14 & 129.3 & 129.3 & 129.0 & 129.0 \\
15 & 128.8 & 128.8 & 128.8 & 128.7 \\
OMe & 64.8 & 64.6 & & \\
\hline
\end{tabular}

${ }^{a}$ Measured in $\mathrm{C}_{6} \mathrm{D}_{6}, 100 \mathrm{MHz}$.

${ }^{b}$ Measured in $\mathrm{CDCl}_{3}, 100 \mathrm{MHz}$.

Table 4. ${ }^{1} \mathrm{H}$ NMR data for compounds 1 and $\mathbf{2}(\delta, \mathrm{ppm}, J, \mathrm{~Hz})$

\begin{tabular}{lllll}
\hline $\mathrm{H}$ & $\mathbf{1 a}^{a}$ & $\mathbf{1 b}^{a}$ & $\mathbf{2 a}^{b}$ & $\mathbf{2 b}^{b}$ \\
\hline 5 & $6.21 \mathrm{q}(1.5)$ & $6.25 \mathrm{q}(1.5)$ & $6.62 \mathrm{q}(1.7)$ & $6.70 \mathrm{q}(1.8)$ \\
6 & $1.64 \mathrm{~d}(1.6)$ & $1.62 \mathrm{~d}(1.6)$ & $2.11 \mathrm{~d}(1.7)$ & $2.12 \mathrm{~d}(1.8)$ \\
8 & $8.36 \mathrm{~d}(15.8)$ & $8.43 \mathrm{~d}(15.8)$ & $7.80 \mathrm{~d}(16.0)$ & $7.80 \mathrm{~d}(16.0)$ \\
9 & $7.60 \mathrm{~d}(15.8)$ & $7.60 \mathrm{~d}(15.8)$ & $7.72 \mathrm{~d}(16.0)$ & $7.73 \mathrm{~d}(16.0)$ \\
11 & $7.45 \mathrm{~m}$ & $7.45 \mathrm{~m}$ & $7.64 \mathrm{~m}$ & $7.64 \mathrm{~m}$ \\
12 & $7.02 \mathrm{~m}$ & $7.02 \mathrm{~m}$ & $7.42 \mathrm{~m}$ & $7.42 \mathrm{~m}$ \\
13 & $7.02 \mathrm{~m}$ & $7.02 \mathrm{~m}$ & $7.42 \mathrm{~m}$ & $7.42 \mathrm{~m}$ \\
14 & $7.02 \mathrm{~m}$ & $7.02 \mathrm{~m}$ & $7.42 \mathrm{~m}$ & $7.42 \mathrm{~m}$ \\
15 & $7.45 \mathrm{~m}$ & $7.45 \mathrm{~m}$ & $7.64 \mathrm{~m}$ & $7.64 \mathrm{~m}$ \\
$\mathrm{OMe}$ & $3.99 \mathrm{~s}$ & $3.94 \mathrm{~s}$ & & \\
$\mathrm{OH}$ & & & $12.11 \mathrm{br} \mathrm{s}$ & $12.23 \mathrm{br} \mathrm{s}$ \\
\hline
\end{tabular}

\footnotetext{
${ }^{a}$ Measured in $\mathrm{C}_{6} \mathrm{D}_{6}, 300 \mathrm{MHz} .{ }^{b}$ Measured in $\mathrm{CDCl}_{3}, 400 \mathrm{MHz}$.
} 

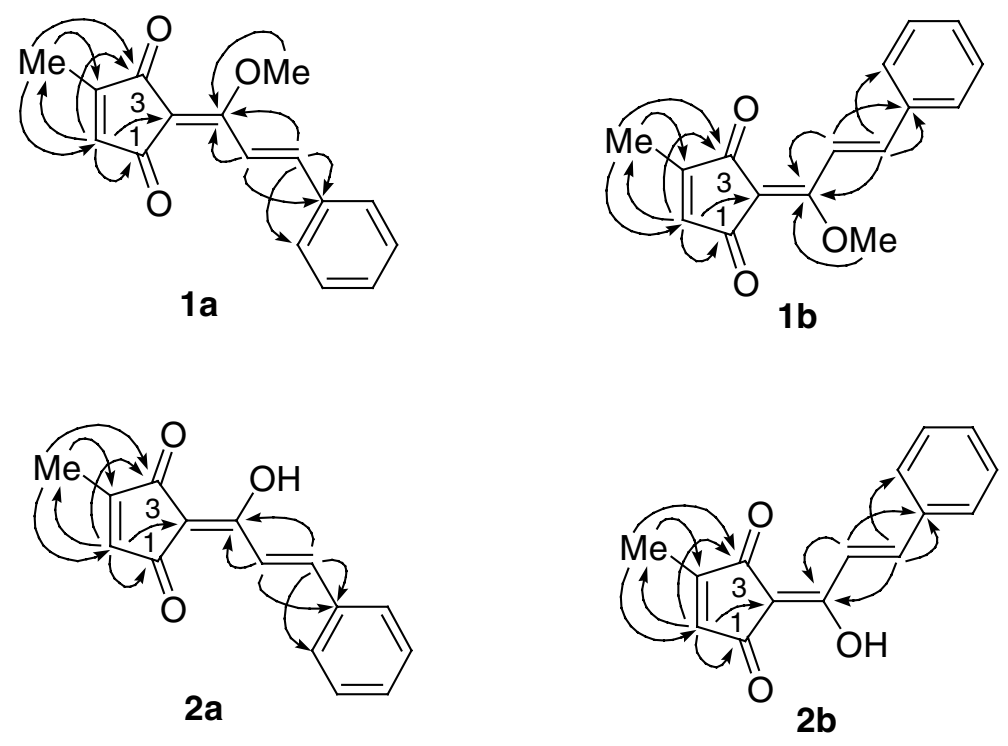

Fig 1. Key HMBC correlations of coruscanones A (1) and B (2) 
Table 5. Antifungal and antibacterial activity of coruscanones A and B $(\mathbf{1}, \mathbf{2})$

\begin{tabular}{clllllll}
\multicolumn{8}{c}{$\left(\mathrm{MIC}^{a} / \mathrm{MFC}^{b}\right.$ or $\left.\mathrm{MBC}^{c}, \mu \mathrm{g} / \mathrm{mL}\right)$} \\
\hline & C. albicans & C. neoformans & A. fumigatus & S. aureus & MRS. aureus & P. aeruginosa & M. intracellulare \\
& ATCC 90028 & ATCC 90113 & ATCC 90906 & ATCC 29213 & ATCC 43300 & ATCC 27853 & ATCC 23068 \\
\hline $\mathbf{1}$ & $0.78 / 1.56$ & 6.25/6.25 & NA $/$ NA & NA/NA & NA/NA & NA/NA & 50/NA \\
$\mathbf{2}$ & 50/NA & NA/NA & NA/NA & 20/NA & NA/NA & NA/NA & 50/NA \\
AMB $^{e}$ & $2.50 / 5.00$ & $2.50 / 2.50$ & $0.63 / 1.25$ & NT $/$ NT & NT/NT & NT/NT & NT/NT \\
CIP $^{s}$ & NT/NT & NT/NT & NT/NT & $0.63 / 0.63$ & $0.63 / 1.25$ & $0.31 / 0.63$ & $1.25 / 5.00$ \\
\hline
\end{tabular}

${ }^{\bar{a}}$ Minimum Inhibitory Concentration. ${ }^{b}$ Minimum Fungicidal Concentration. ${ }^{c}$ Minimum Bactericidal Concentration. ${ }^{d}$ Not active. ${ }^{e}$ Amphotericin B

${ }^{f}$ Not tested. ${ }^{8}$ Ciprofloxacin.

Table 6. In vitro cytotoxicity of coruscanones A and B (1,2) $\left(\mathrm{IC}_{50}, \mu \mathrm{g} / \mathrm{mL}\right)$

(Assay method is published in our previous paper, Muhammad, I. et al., J. Nat. Prod. 2003, 66, 962.)

\begin{tabular}{clllll}
\hline & SK-MEL $^{a}$ & $\mathrm{~KB}^{b}$ & BT-549 $^{c}$ & SK-OV-3 & Vero $^{e}$ \\
\hline $\mathbf{1}$ & 2.8 & 5.9 & 6.1 & 6.8 & 5.3 \\
$\mathbf{2}$ & 9.5 & 7.4 & 5.7 & 6.2 & $\mathrm{NC}^{f}$ \\
Doxorubicin & 1.5 & 1.2 & 1.9 & 1.9 & $\mathrm{NC}$ \\
Amphotericin B & 5.7 & $\mathrm{NC}$ & 1.5 & $\mathrm{NC}$ & 6.5 \\
\hline
\end{tabular}

${ }^{a}$ Malignant melanoma, human. ${ }^{b}$ Epidermoid carcinoma, oral, human.

${ }^{c}$ Ductal carcinoma, breast, human. ${ }^{d}$ Ovary carcinoma, human;

${ }^{e}$ Kidney, African green monkey. ${ }^{f}$ Not cytotoxic up to $10 \mu \mathrm{g} / \mathrm{mL}$. 
Cinnamoyl methylenetriphenylphosphorane (4) (according to the procedure described in Clemo, N. G.;

Gedge, D. R.; Pattenden, G. J. Chem. Soc., Perkin Trans. 1 1981, 1448-1453 and the literature cited therein)
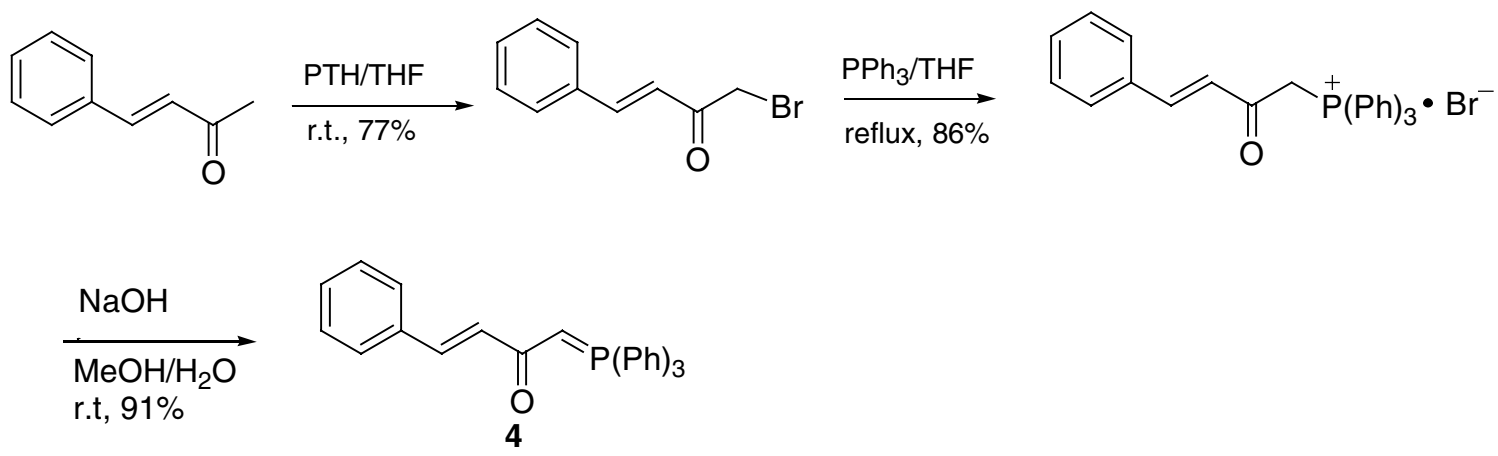

To a solution of styryl methyl ketone $(5.0 \mathrm{~g}, 34.2 \mathrm{mmol})$ in dry THF $(80 \mathrm{~mL})$ at room temperature under nitrogen was slowly added a solution of pyrrolidone hydrotribromide (20.3 g, $40.9 \mathrm{mmol})$ in dry THF $(120 \mathrm{~mL})$ in $1 \mathrm{hr}$. The mixture was continued to stir at room temperature for $24 \mathrm{hrs}$. Excess pyrrolidone hydrotribromide was removed by filtration. The filtrate was concentrated to dryness. The resulting residue was dissolved in $\mathrm{Et}_{2} \mathrm{O}$, washed with brine and dried $\left(\mathrm{Na}_{2} \mathrm{SO}_{4}\right)$. Removal of the solvents afforded a crude product, which was chromatographed on silica gel eluting with $\mathrm{Et}_{2} \mathrm{O}$-hexanes (1:6) to give bromomethyl styryl ketone $\left(5.91 \mathrm{~g}, 77 \%\right.$ ) as colorless crystals (from $\left.\mathrm{Et}_{2} \mathrm{O}\right),{ }^{1} \mathrm{H} \mathrm{NMR}\left(400 \mathrm{MHz}, \mathrm{CDCl}_{3}\right) \delta$ $7.70(1 \mathrm{H}, \mathrm{d}, J=16.0 \mathrm{~Hz}, \beta-\mathrm{CH}=), 7.58(2 \mathrm{H}, \mathrm{m}, \mathrm{Ph}), 7.43(3 \mathrm{H}, \mathrm{m}, \mathrm{Ph}), 6.95(1 \mathrm{H}, \mathrm{d}, J=16.0 \mathrm{~Hz}, \alpha-\mathrm{CH}=)$, $4.11\left(2 \mathrm{H}, \mathrm{s}, \mathrm{CH}_{2} \mathrm{Br}\right) ;{ }^{13} \mathrm{C} \mathrm{NMR}\left(100 \mathrm{MHz} \mathrm{CDCl}_{3}\right) \delta 191.1(\mathrm{~s}, \mathrm{C}=\mathrm{O}), 145.4(\mathrm{~d}, \beta-\underline{\mathrm{CH}}=), 134.0(\mathrm{~s}, \mathrm{Ph})$, 131.2 (d, Ph), 129.1 (2C, d, Ph), 128.7 (2C, d, Ph), 122.3 (d, $\alpha-\underline{C} H=), 33.3$ (t, $\underline{\left.\mathrm{CH}_{2} \mathrm{Br}\right) .}$

To a refluxed solution of triphenylphosphine $(5.84 \mathrm{~g}, 22.3 \mathrm{mmol})$ in dry THF (25 $\mathrm{mL})$ under nitrogen was slowly added bromomethyl styryl ketone $(5.0 \mathrm{~g}, 22.3 \mathrm{mmol}$, in $25 \mathrm{ml} \mathrm{THF})$. The reaction 
mixture was refluxed for 2 hrs. After cooling, the white precipitate was collected by filtration and washed with $\mathrm{Et}_{2} \mathrm{O}$. Crystallization of the product with methanol gave triphenyl cinnamoylmethyl phosphonium bromide $(9.3 \mathrm{~g}, 86 \%)$ as colorless needles, ${ }^{1} \mathrm{H}$ NMR $\left(400 \mathrm{MHz}, \mathrm{DMSO}-d_{6}\right) \delta 8.02(1 \mathrm{H}, \mathrm{d}, J=16.0 \mathrm{~Hz}, \beta-$ $\mathrm{CH}=), 7.87,7.76,7.49(20 \mathrm{H}, \mathrm{m}, \mathrm{Ph}), 7.00(1 \mathrm{H}, \mathrm{d}, J=16.0 \mathrm{~Hz}, \alpha-\mathrm{CH}=), 6.07\left(2 \mathrm{H}, \mathrm{d}, J=13.2 \mathrm{~Hz}, \mathrm{CH}_{2} \mathrm{Br}\right)$.

To a suspension of the above phosphonium salts $(8.1 \mathrm{~g}, 16.7 \mathrm{mmol})$ in water $(100 \mathrm{~mL})$ and methanol $(100 \mathrm{~mL})$ was added a solution of sodium hydroxide $(1.3 \mathrm{~g})$ in water $(15 \mathrm{~mL})$. The mixture was stirred at room temperature overnight. After evaporation of methanol, the suspension was extracted with chloroform. The organic layer was washed with brine, dried $\left(\mathrm{Na}_{2} \mathrm{SO}_{4}\right)$, and concentrated to dryness. Crystallization of the residue with $\mathrm{Et}_{2} \mathrm{O}$ gave corresponding phosphorane $4(6.2 \mathrm{~g}, 91 \%)$ as yellow crystals, ${ }^{1} \mathrm{H}$ NMR $\left(400 \mathrm{MHz}, \mathrm{CDCl}_{3}\right) \delta 7.53,7.47,7.31,7.24(21 \mathrm{H}, \mathrm{m}), 6.94(1 \mathrm{H}, \mathrm{d}, J=16.0 \mathrm{~Hz}, \alpha-\mathrm{CH}=), 4.07$ $(1 \mathrm{H}$, br d, $J=21.2 \mathrm{~Hz}, \mathrm{CH}=\mathrm{P}) ;{ }^{13} \mathrm{C} \mathrm{NMR}\left(100 \mathrm{MHz}, \mathrm{CDCl}_{3}\right) \delta 182.8(\mathrm{C}=\mathrm{O}), 136.8(\mathrm{~d}, \beta-\underline{\mathrm{CH}}=), 133.9$, $133.2,133.1,132.3,129.1,129.0,128.6,128.2,127.5,127.1,126.6$.

E-4-cinnamoylmethylidene-2-methylbut-2-en-4-olide (5): A solution of 2-methylmaleic anhydride (3) $(1.8 \mathrm{~g}, 16.1 \mathrm{mmol})$ in benzene $(10 \mathrm{~mL})$ was added to a refluxed solution of $\mathbf{4}(5.5 \mathrm{~g}, 13.5 \mathrm{mmol})$ in benzene $(50 \mathrm{~mL})$ under nitrogen. After refluxing for $6 \mathrm{hrs}$, TLC indicated complete consumption of $\mathbf{4}$. The reaction mixture was evaporated to dryness, and the residue was chromatographed on silica gel using hexaneacetone (4:1) to yield E-4-cinnamoylmethylidene-2-methylbut-2-en-4-olide (5) (2.35 g, 73\%) as yellow needles [from chloroform-methanol (1:1)]: mp 160-161 Co, UV (MeOH), $\lambda_{\max }(\log \varepsilon) 208$ (4.18), 238 (3.98), 334 (4.50) nm; IR (KBr) $v_{\max }, 1770,1678,1627,1588,1451,1375,1209,1098,1039,990,911$, 877, 754, 698, 545; ${ }^{1} \mathrm{H}$ NMR (400 MHz, $\left.\mathrm{CDCl}_{3}\right) \delta 8.10(1 \mathrm{H}, \mathrm{s}, \mathrm{H}-3), 7.64\left(1 \mathrm{H}, \mathrm{d}, J=16.0 \mathrm{~Hz}, \mathrm{H}-4^{\prime}\right), 7.59$ $\left(2 \mathrm{H}, \mathrm{m}, \mathrm{H}-6^{\prime}, 10^{\prime}\right), 7.42\left(3 \mathrm{H}, \mathrm{m}, \mathrm{H}-7^{\prime}, 8^{\prime}, 9^{\prime}\right), 6.88\left(1 \mathrm{H}, \mathrm{d}, J=16.0 \mathrm{~Hz}, \mathrm{H}-3^{\prime}\right), 6.48\left(1 \mathrm{H}, \mathrm{s}, \mathrm{H}-1^{\prime}\right), 2.11(3 \mathrm{H}, \mathrm{s}$, Me-2); ${ }^{13} \mathrm{C}$ NMR (100 MHz, $\left.\mathrm{CDCl}_{3}\right) \delta 188.3\left(\mathrm{~s}, \mathrm{C}-2^{\prime}\right), 169.2$ (s, C-1), 159.2 (s, C-4), 144.0 (d, C- 
4'), 137.0 (d, C-3), 136.4 (s, C-2), 134.3 (s, C-5'), 131.0 (d, C-8'), 129.1 (2C, d, C-6' $\left.10^{\prime}\right), 128.6(2 \mathrm{C}, \mathrm{d}, \mathrm{C}-$ $\left.7^{\prime}, 9^{\prime}\right), 127.5\left(\mathrm{~d}, \mathrm{C}-3^{\prime}\right), 106.0\left(\mathrm{~d}, \mathrm{C}-1^{\prime}\right), 11.2$ (q, Me-2) (NMR assignments were based on 2D HMBC and NOESY experiments); HRESIMS $m / z[\mathrm{M}+\mathrm{H}]^{+} 241.0841$ (calcd for $\mathrm{C}_{15} \mathrm{H}_{13} \mathrm{O}_{3}, 241.0859$ ), $[\mathrm{M}+\mathrm{Na}]^{+}$ 263.0659 (calcd for $\mathrm{C}_{15} \mathrm{H}_{12} \mathrm{O}_{3} \mathrm{Na}, 263.0678$ ), $[\mathrm{M}+\mathrm{K}]^{+} 279.0400$ (calcd for $\mathrm{C}_{15} \mathrm{H}_{12} \mathrm{O}_{3} \mathrm{~K}, 279.0418$ ).

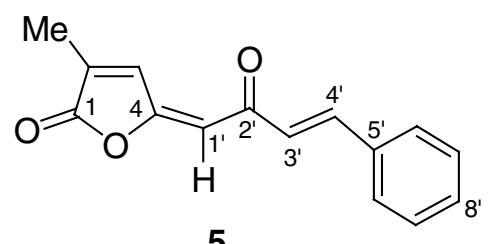

5

Coruscanone B (2): A solution of 5 (1.95 g, $8.13 \mathrm{mmol})$ in dry $\mathrm{MeOH}(60 \mathrm{~mL})$ was added to a solution of $\mathrm{NaOMe}(4.41 \mathrm{~g}, 81.7 \mathrm{mmol})$ in $\mathrm{MeOH}(25 \mathrm{~mL})$, and the resulting orange-colored solution was stirred at room temperature for $3 \mathrm{hr}$ and then poured onto ice-water $(150 \mathrm{~mL})$ and acidified to $\mathrm{pH} 1.0$ with $2 \mathrm{M} \mathrm{HCl}$. The methanol was removed by evaporation, and the suspension was extracted with $\mathrm{Et}_{2} \mathrm{O}$. Evaporation of the dried extracts gave the crude product which was purified by crystallization with $\mathrm{MeOH}$ to yield coruscanone B (2) as yellow needles $(1.21 \mathrm{~g}, 62 \%)$, mp. $125{ }^{\circ} \mathrm{C}$. Note: The NMR spectra indicate this synthetically crystalline compound possesses predominant isomer $2 \boldsymbol{b}$ (refer to its ${ }^{1} \mathrm{H}$ NMR spectrum on S14). Similar to naturally occurring coruscanone B, isomer $2 \boldsymbol{b}$ could rapidly convert to $2 \boldsymbol{a}$ in $\mathrm{CDCl}_{3}(\mathrm{refer}$ to its ${ }^{1} H$ NMR spectrum on S15).

Coruscanone A (1): To a solution of $2(500 \mathrm{mg})$ in dry acetone $(50 \mathrm{~mL})$ under argon was added $\mathrm{K}_{2} \mathrm{CO}_{3}$ $(3.0 \mathrm{~g})$. After refluxing for $5 \mathrm{~min}, \mathrm{Me}_{2} \mathrm{SO}_{4}(2.2 \mathrm{~mL})$ was added to the deep yellow-colored solution, and the mixture was continued to reflux for 30 minutes. The $\mathrm{K}_{2} \mathrm{CO}_{3}$ was removed by filtration. The filtrate was concentrated to dryness, and the residue was subjected to reversed phase RP-18 column chromatography using aqueous $\mathrm{MeOH}$. The yellow band was eluted by $70 \% \mathrm{MeOH}$, and evaporation of the solvent give coruscanone A (1) (493 mg, 93\%) as a yellow powder whose NMR spectra are identical to those of natural one (refer to the ${ }^{1} \mathrm{H}$ NMR spectra on S12 and S11). 


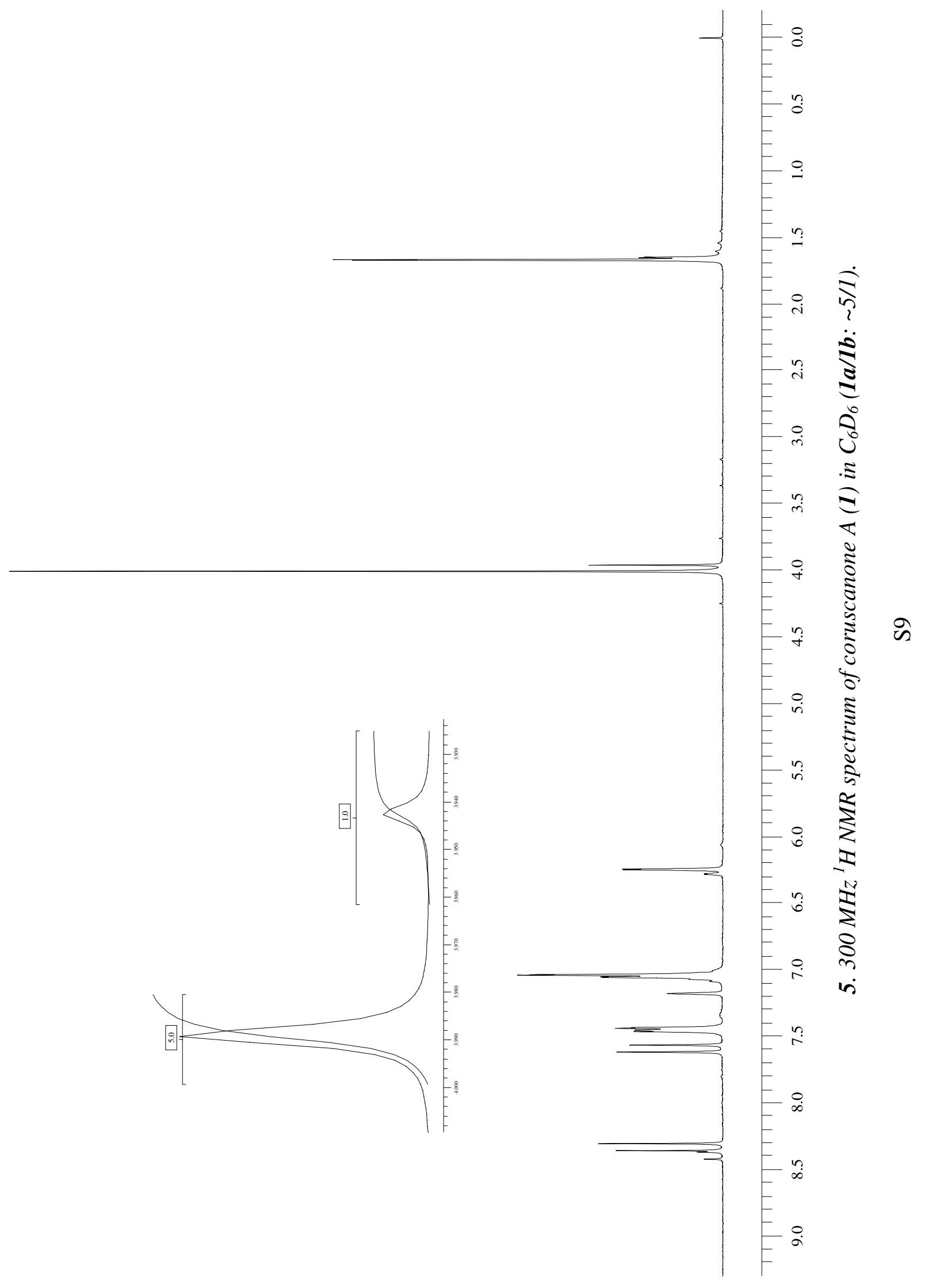




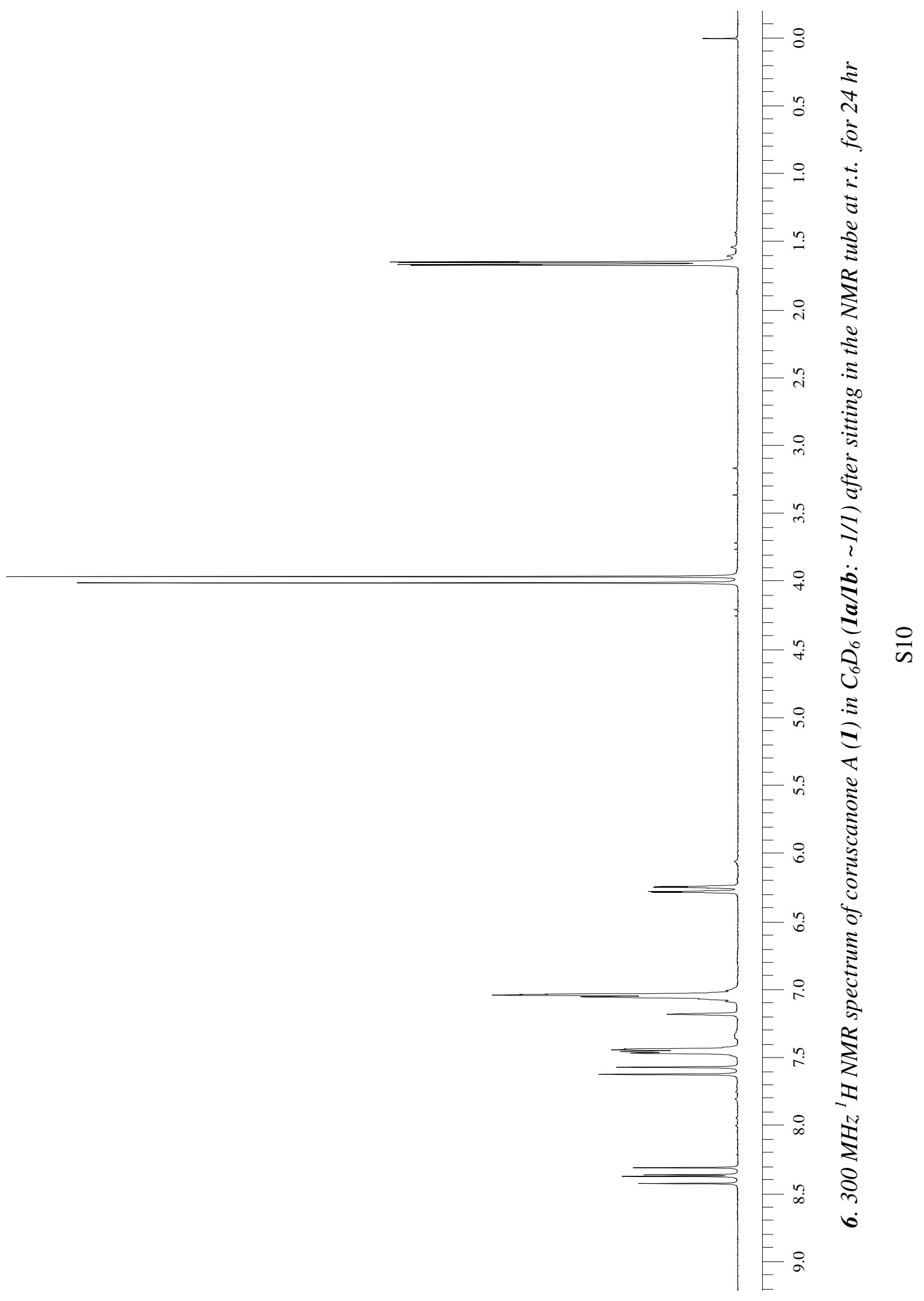




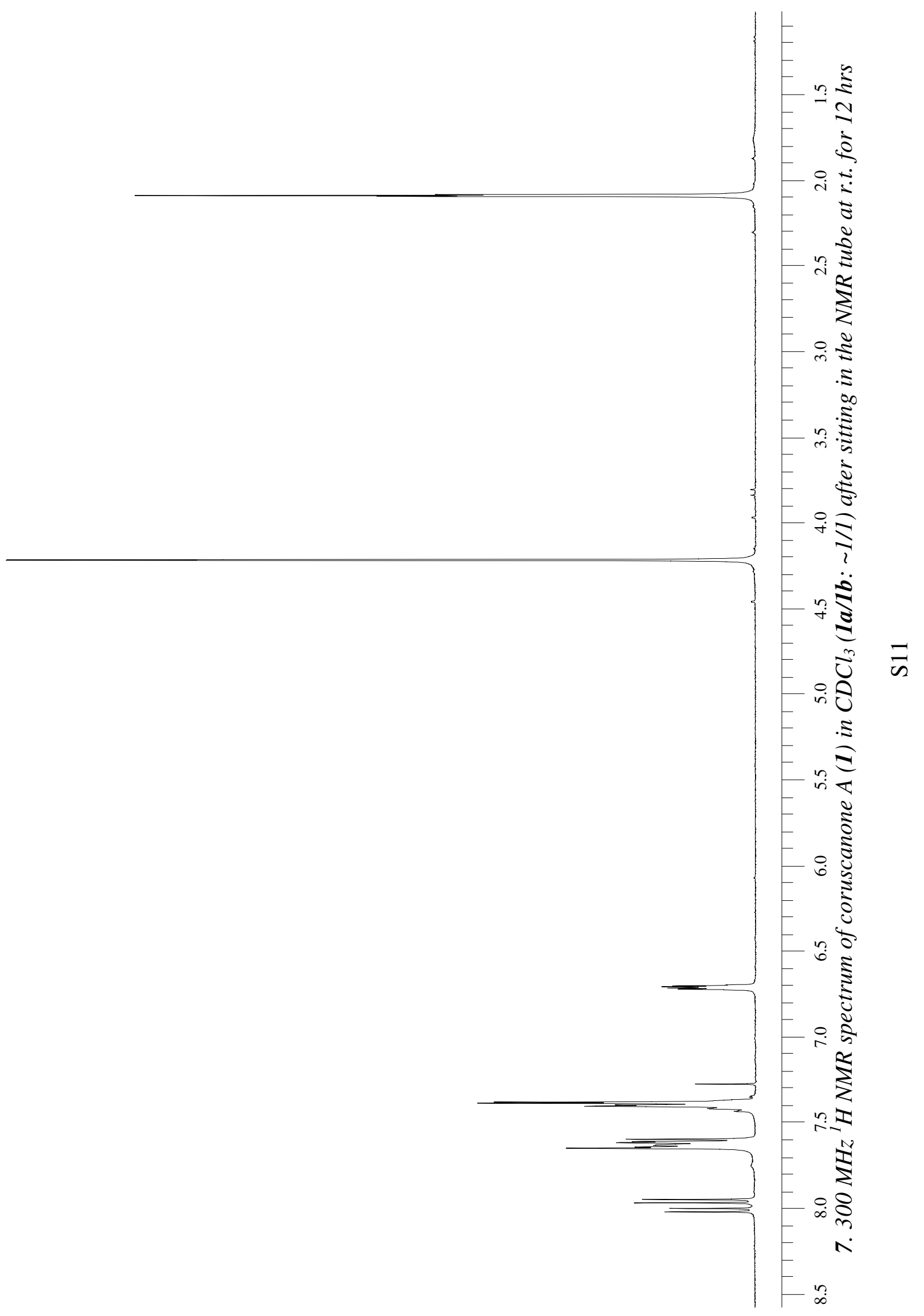




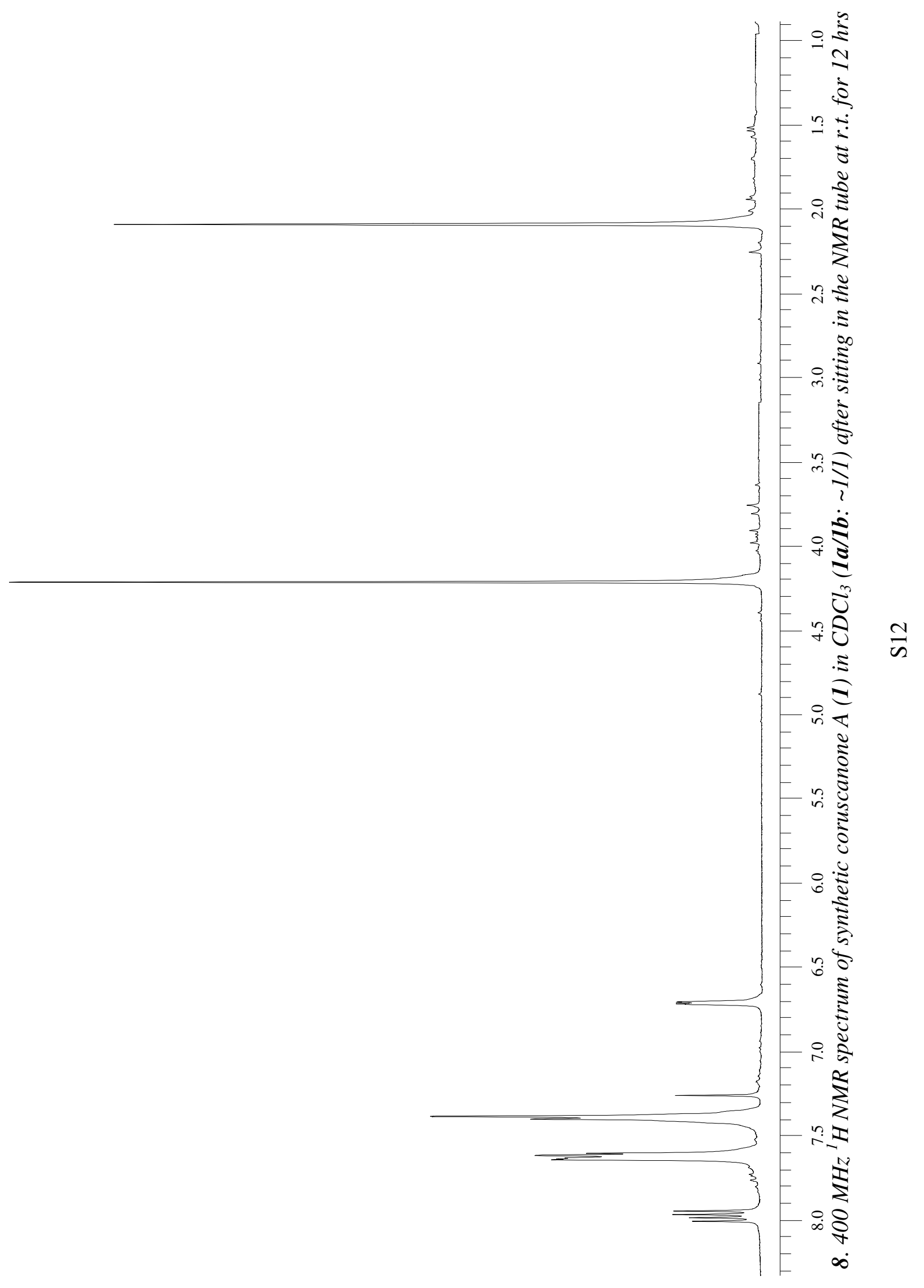




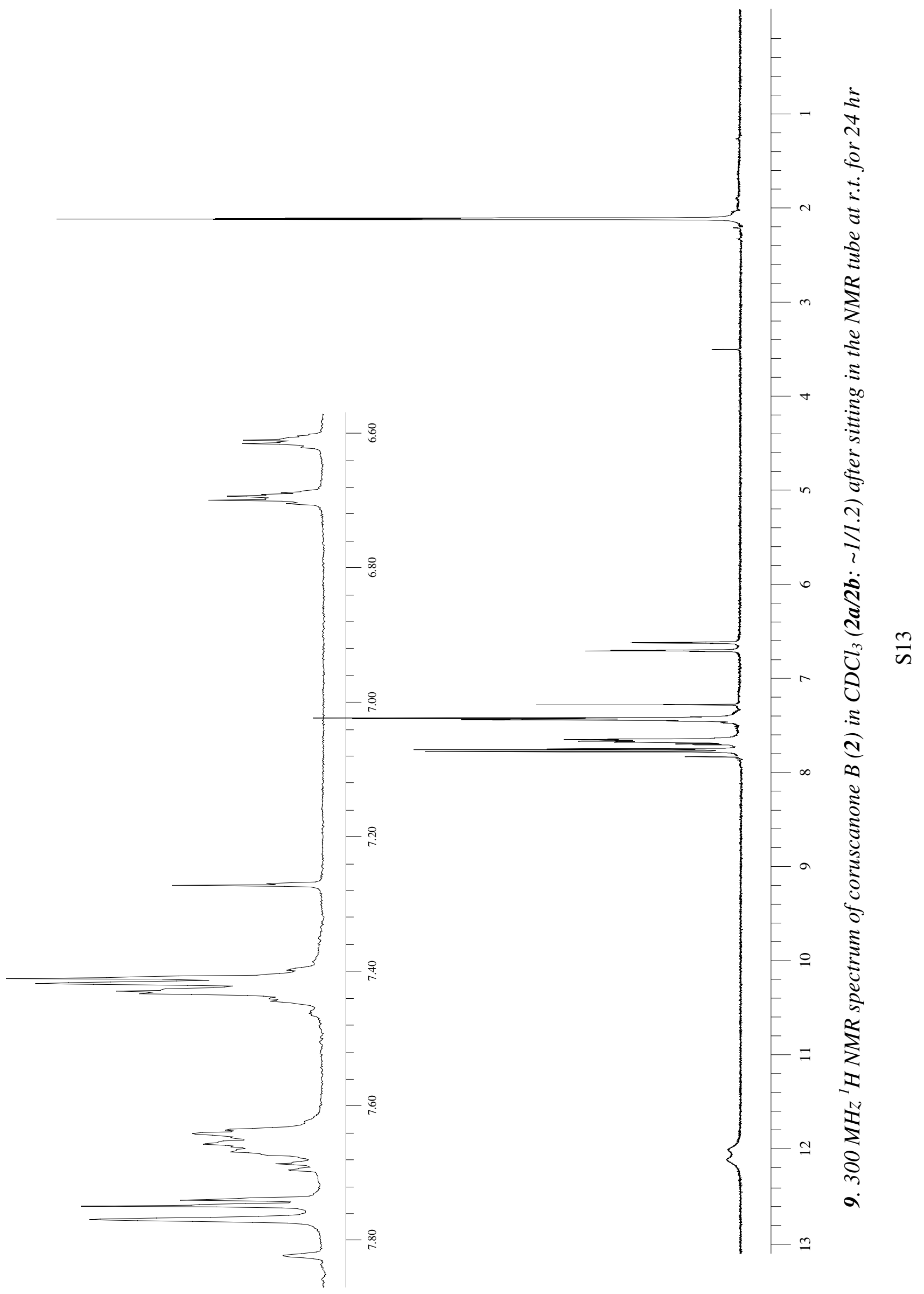




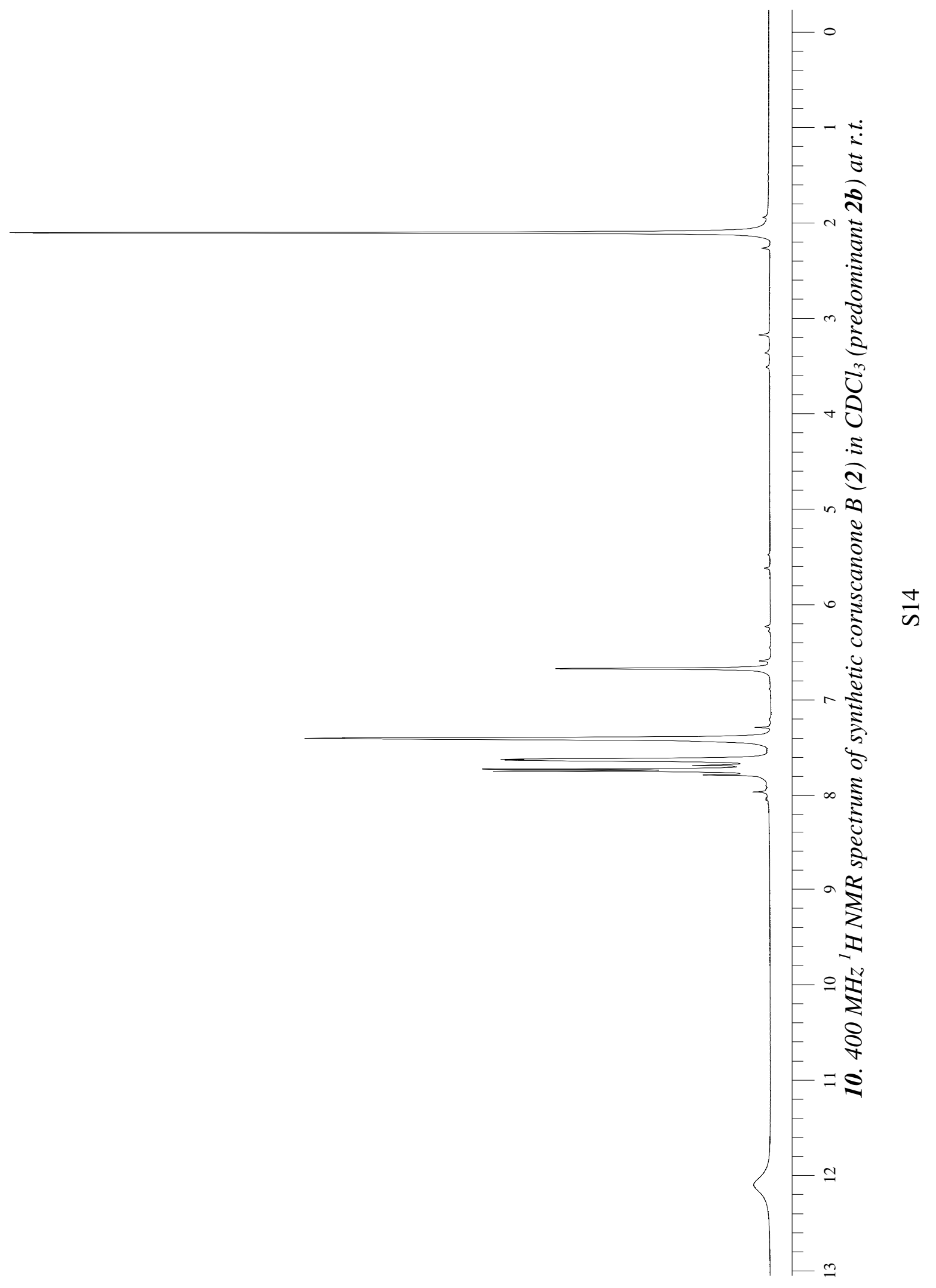




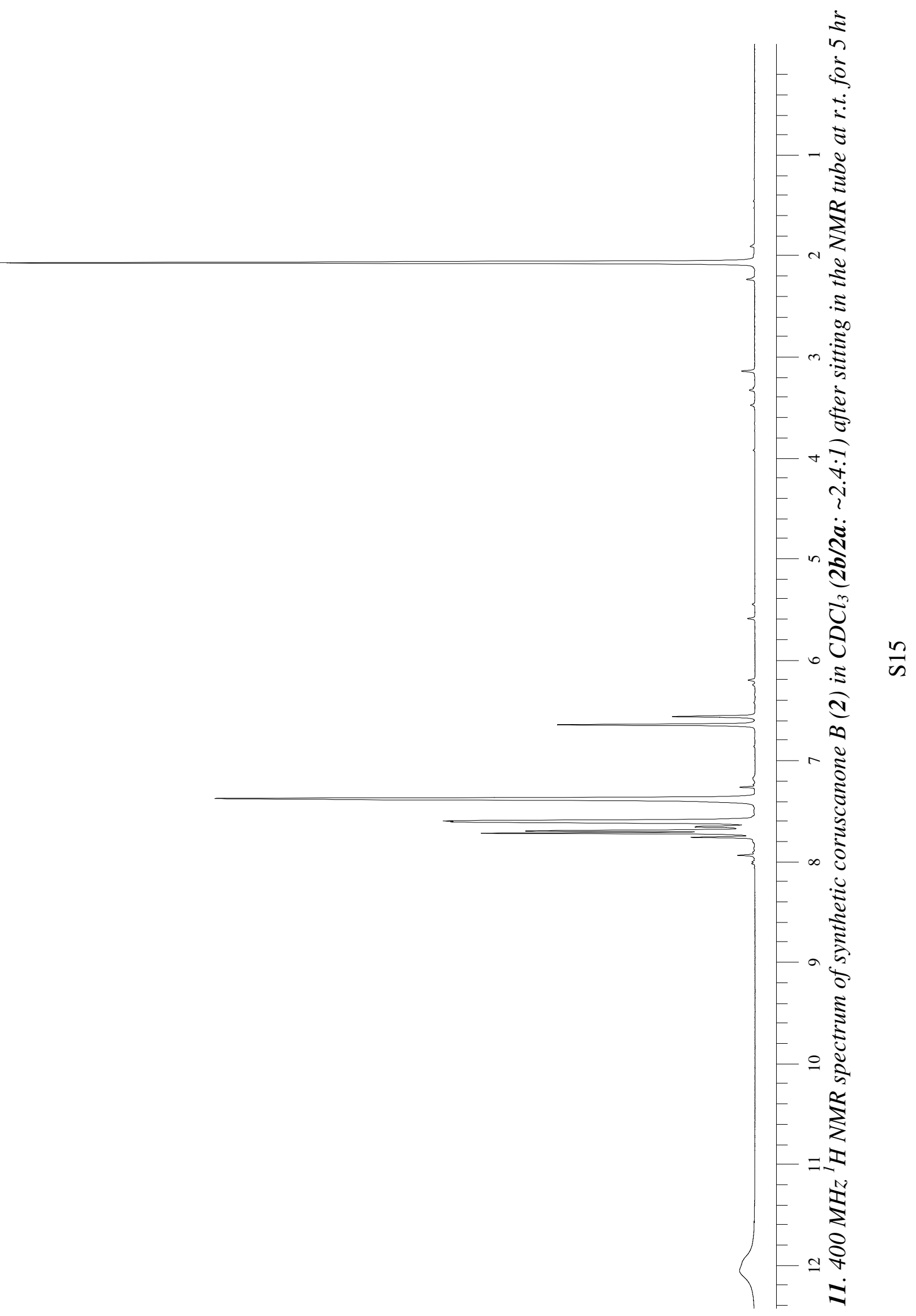




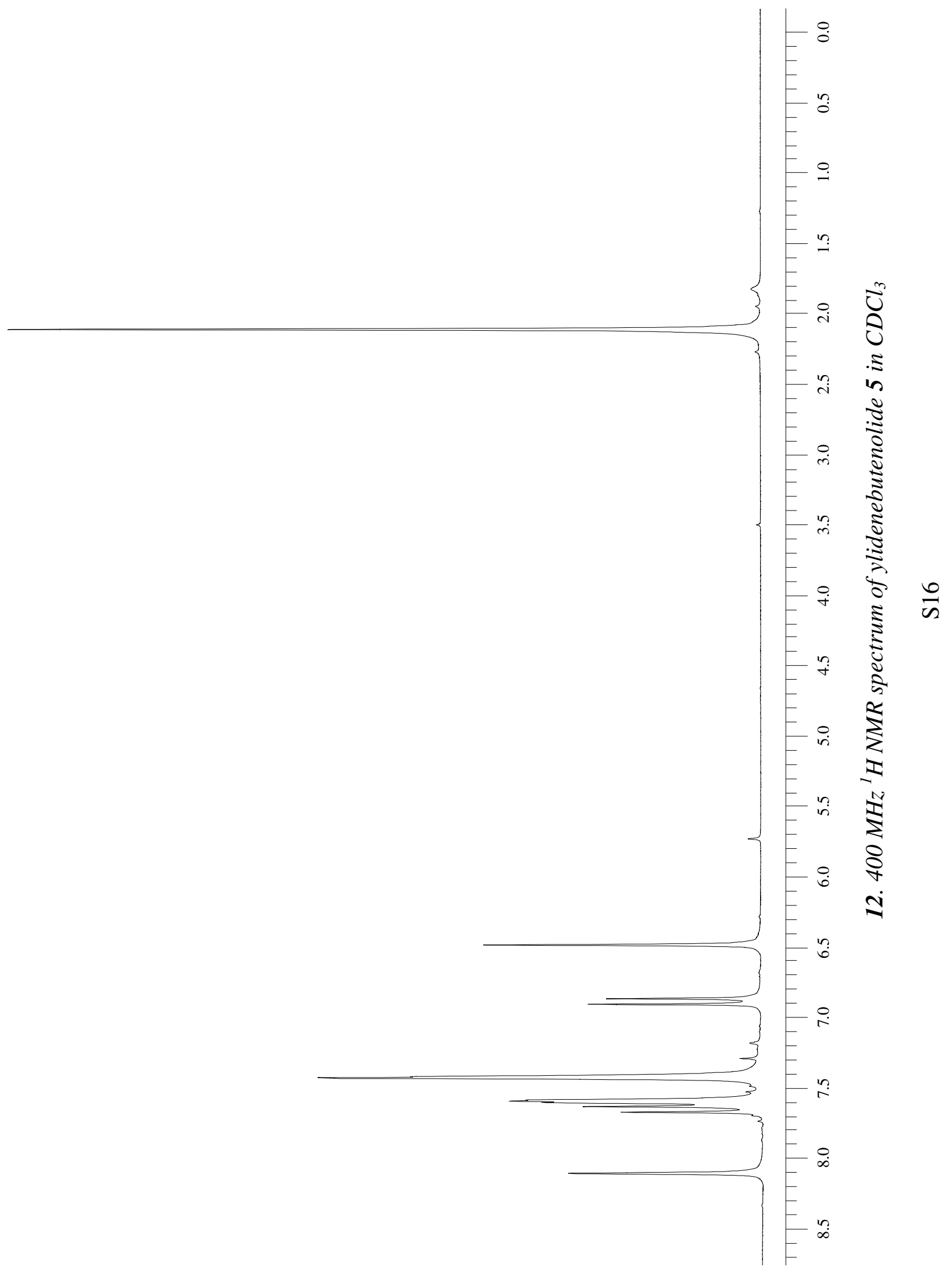

